\title{
A physical model of differential Mueller matrix for depolarizing uniform media
}

\author{
Vincent Devlaminck \\ LAGIS - UMR CNRS 8219, Université Lille 1, Sciences et Technologies - Villeneuve d'Ascq, France \\ Corresponding author: vincent.devlaminck@univ-lille1.fr
}

\section{INTRODUCTION}

Based on a layered-medium interpretation, Jones introduced the concept of "N-matrices" in the seventh paper of his series [1]. He also derived a differential equation governing the evolution of the state of polarization for a totally polarized light beam propagating through a linear and non depolarizing medium:

$$
\frac{\mathrm{d} \mathbf{E}}{\mathrm{d} z}=\mathbf{N} \mathbf{E}
$$

$\mathbf{E}$ is the Jones vector associated to the two dimensional electric field vector propagating along the $z$ direction.

Later, Azzam extended this approach [2] to the partially polarized light propagating through non depolarizing media and found that the Stokes vector of the light obeys the equation:

$$
\frac{\mathrm{d} \mathbf{S}}{\mathrm{d} z}=\mathbf{m}_{N D} \mathbf{S}
$$

where $\mathbf{m}_{N D}$ is termed the differential Mueller matrix and $\mathbf{S}$ stands for the Stokes vector of the light at distance $z$ into the medium. Azzam also derived the relations between the entries of $\mathbf{N}$ and $\mathbf{m}_{N D}$ differential matrices for non depolarizing media. However, the formal relation between these both matrices was formulated by Barakat [3]. From the concept of exponential versions of the Mueller-Jones matrices and properties of the Kronecker product of matrices, Barakat established the following relation:

$$
\mathbf{m}_{N D}=\Lambda^{\dagger}\left(\mathbf{N} \otimes \mathbf{I}+\mathbf{I} \otimes \mathbf{N}^{*}\right) \Lambda
$$

Where $\mathbf{I}$ is the identity matrix, $\uparrow$ and $*$ stand for a Hermitian and complex conjugate respectively and

$$
\Lambda=\frac{1}{\sqrt{2}}\left[\begin{array}{cccc}
1 & 1 & 0 & 0 \\
0 & 0 & 1 & -\mathrm{i} \\
0 & 0 & 1 & \mathrm{i} \\
1 & -1 & 0 & 0
\end{array}\right]
$$

It is worth noticing that in his paper, Azzam derived Eq. (2) for non depolarizing media only but extended the scope of this result to depolarizing media by noting:

"Polarizing medium has the following properties: (1) all diagonal elements are equal, and (2) off-diagonal elements symmetrically located with respect to the main diagonal are either equal or differ only in sign. ... Breaking any of these symmetry conditions produces a matrix $\mathrm{m}$ that must correspond to a depolarization phenomenon, or else a matrix that is 
entirely nonphysical." However, he did not provide a general method and the result for depolarizing media was just illustrated by an example.

Although a generalization of Azzam's differential matrix formalism to depolarizing Mueller matrices was proposed by Ossikovski [4] and Ortega-Quijano \& al. [5-6] as:

$$
\mathbf{m}=\left[\begin{array}{cccc}
d_{0} & d_{1}+d_{7} & d_{2}+d_{8} & d_{3}+d_{9} \\
d_{1}-d_{7} & d_{0}-d_{13} & d_{12}+d_{6} & d_{11}-d_{5} \\
d_{2}-d_{8} & d_{12}-d_{6} & d_{0}-d_{14} & d_{10}+d_{4} \\
d_{3}-d_{9} & d_{11}+d_{5} & d_{10}-d_{4} & d_{0}-d_{15}
\end{array}\right]
$$

In Ref. [4], Ossikovski provides a physical interpretation of this symmetry breaking in the matrix $\mathbf{m}$. He proposes to consider the $d_{0-6}$ parameters as mean values of the non-depolarizing properties $\left(\mathbf{m}_{N D}\right.$ part) and $d_{7-15}$ as their respective uncertainties resulting from the depolarization $\left(\mathbf{m}_{D}\right.$ part). The elementary optical non-depolarizing properties are [1] the amplitude or isotropic absorption $\left(d_{0}\right)$, the linear dichroism along the $\mathrm{x}-\mathrm{y}$ laboratory axes $\left(d_{1}\right)$, the linear dichroism along the $45^{\circ}$ axes $\left(d_{2}\right)$, the circular dichroism $\left(d_{3}\right)$, the linear birefringence along the $\mathrm{x}-\mathrm{y}$ axes $\left(d_{4}\right)$, the linear birefringence along the $45^{\circ}$ axes $\left(d_{5}\right)$ and the circular birefringence $\left(d_{6}\right) . d_{13-15}$ are the anisotropic absorptions coefficients along the $\mathrm{x}-\mathrm{y}, 45^{\circ}$ and circular axes respectively.

Under this approach, differential matrix formalism in polarization optics connects the Mueller matrix $\mathrm{M}(z)$ at distance $z$ into the medium to its spatial derivative along the light propagation direction. For a homogeneous medium, the solution of this differential equation is given by:

$$
\mathbf{M}(z)=\exp (z \mathbf{m})
$$

The Mueller matrix logarithm $\mathbf{L}$ is then proportional to the differential matrix $\mathbf{m}$ and can be decomposed in two terms $\mathbf{L}_{m}$ and $\mathbf{L}_{u}$ related to $\mathbf{m}_{N D}$ and $\mathbf{m}_{D}$ respectively with:

$$
\mathbf{L}=\ln (\mathbf{M})=\mathbf{L}_{m}+\mathbf{L}_{u}
$$

$\mathbf{L}_{m}$ and $\mathbf{L}_{u}$ are derived from $\mathbf{L}$ by [3]:

$$
\begin{aligned}
& \mathbf{L}_{m}=\frac{1}{2}\left(\mathbf{L}-\mathbf{G L}^{\mathrm{T}} \mathbf{G}\right) \\
& \mathbf{L}_{u}=\frac{1}{2}\left(\mathbf{L}+\mathbf{G L}^{\mathrm{T}} \mathbf{G}\right)
\end{aligned}
$$

where $\mathbf{G}=\operatorname{diag}(1,-1,-1,-1)$ is the Minkowski metric.

It is worth noticing that the Mueller matrix roots decomposition was introduced in [7-8] to provide an order-independent description of polarization properties. Nevertheless, the roots and the Mueller matrix logarithm decompositions are equivalent as demonstrated by Ossikovski [9] although obtained from different mathematical procedures.

However, $\mathbf{m}$ formulation of Eq. (5) leads to various comments.

As noted by Germer [10]: “... with the exception of $d_{13-15}$, setting any one of these depolarizing coefficients to non-zero value will result in an invalid Mueller matrix." So there must be a relationship between the off-diagonal coefficients $d_{7-12}$ physically representing the uncertainties of the respective elementary polarization properties $d_{1-6}$ and the diagonal anisotropic depolarizations $\mathrm{d}_{13-15}$. In order to solve this problem Germer [10] proposes a very interesting approach to ensure that the parameterization will only lead to physical Mueller matrices. Based on three types of depolarization a new parameterization is proposed. Each of these depolarization types is related to a direction in Poincaré space, yielding a total of nine adjustable parameters. The off-diagonal depolarizing coefficients of $\mathbf{m}$ are function of these nine parameters and are no more independent.

Nevertheless, this approach suffers from two main drawbacks: 
a) Hypothesis is only valid for the definition of Mueller matrix as a matrix which transforms the space of valid Stokes vectors into a subspace of valid Stokes vectors but is not valid for the correct definition of Mueller matrix as a convex sum of MuellerJones [11].

b) The obtained parameterization does not describe the full set of physically admissible differential matrices (diagonal matrices with different entries on the diagonal are not possible with this parameterization for instance).

Nevertheless, how the depolarizing part of a physical differential Mueller matrix can be correctly parameterized has been explained in recent articles [12-13]. It has been first demonstrated [12] that diagonal differential matrices are always given by:

$$
\left[\begin{array}{cccc}
k_{1}+k_{2}+k_{3} & 0 & 0 & 0 \\
0 & k_{1}-k_{2}-k_{3} & 0 & 0 \\
0 & 0 & k_{2}-k_{1}-k_{3} & 0 \\
0 & 0 & 0 & k_{3}-k_{1}-k_{2}
\end{array}\right]
$$

Where $\left(k_{1}, k_{2}, k_{3}\right)$ parameters have to be positive in order to lead to physical Mueller matrices (as a convex sum of MuellerJones matrices).

The general expression of this depolarizing part is then given by [13]:

$$
\mathbf{L}_{\mathrm{u}}=\mathbf{A} \mathbf{m}^{(\mathrm{i})} \mathbf{A}^{-1}
$$

where $\mathbf{A} \in S O(3,1)$ whose generators are $\mathbf{G}_{1-6}$. Then $\mathbf{A}$ can be parameterized as $\mathbf{A}=\mathrm{e}^{\mathbf{G}}$ with $\mathbf{G}=\alpha \mathbf{G}_{1}+\beta \mathbf{G}_{2}+\gamma \mathbf{G}_{3}+\mu \mathbf{G}_{4}+v$ $\mathbf{G}_{5}+\rho \mathbf{G}_{6}$. So $\mathbf{m}_{\boldsymbol{D}}=\mathrm{e}^{\mathbf{G}} \mathbf{m}^{(\mathrm{i})} \mathrm{e}^{-\mathbf{G}}$. $\mathbf{m}^{(\mathrm{i})}$ is either

$$
\begin{aligned}
& \mathbf{m}^{(1)}=\operatorname{diag}\left(k_{1}+k_{2}+k_{3}, k_{1}-k_{2}-k_{3},\right. \\
& \left.k_{2}-k_{1}-k_{3}, k_{3}-k_{1}-k_{2}\right) \\
& \text { or } \\
& \mathbf{m}^{(2)}=\left[\begin{array}{cccc}
0.5\left(k_{4}+k_{1}\right) & 0.5 k_{4} & 0 & 0 \\
-0.5 k_{4} & -0.5\left(k_{4}-k_{1}\right) & 0 & 0 \\
0 & 0 & -0.5 k_{1} & 0 \\
0 & 0 & 0 & -0.5 k_{1}
\end{array}\right]
\end{aligned}
$$

Regardless to Eq. (11) of [13] an overall isotropic absorption factor $-0.5\left(\mathrm{k}_{1}+\mathrm{k}_{4}\right)$ is introduced in $\mathbf{m}^{(2)}$ in order to be consistent with the present notation where left upper corner entry of the matrix can be non zero. This isotropic absorption coefficient can always be removed by subtracting $0.5\left(\mathrm{k}_{1}+\mathrm{k}_{4}\right) \mathbf{I}$ from the initial matrix and has no effect on the values of the rest of the properties.

In order to lead to physical Mueller matrices defined as a convex sum of Mueller-Jones matrices, the $\left(k_{1}, k_{2}, k_{3}\right)$ or $\left(k_{1}, k_{4}\right)$ parameters have to be positive while free of constraint are the six other parameters related to $\mathbf{A}$.

Although very relevant, nothing in the proposed model of Eq. (5) can explain why the diagonal coefficients should check some relationships described in Ref [12] and Eq. (9) or why the mean values of the non-depolarizing properties ( $\mathbf{m}_{N D}$ part) and their respective uncertainties resulting from the depolarization are not independent, as is shown in Ref $[10,13]$.

Although these results were also experimentally confirmed [14], it remains to explain the physical basis and link the various properties of these differential matrices to underlying physical phenomena. This is exactly the question that we address in the following sections of this article.

\section{LAYER MEDIUM APPROACH}




\section{A. Non depolarizing medium}

Addressing this question may be founded on the similar approach to the layered-medium interpretation proposed by Jones in the seventh paper of his series [1]. If a very thin section of a medium is considered from a nondepolarizing element, the resulting Mueller matrix $\mathbf{M}^{\mathrm{e}}$ differs only slightly from the Identity matrix. This elementary matrix can be considered as a sandwich of six thin laminae related to elementary optical properties of the medium ( 3 for dichroïsm, 3 for birefringence and one for amplitude absorption). Let the corresponding thicknesses and generators associated to these laminae named $\tau_{\mathrm{i}}$ and $\mathbf{G}_{\mathrm{i}}$ respectively. The elementary Mueller matrix associated to one of these laminae can be written as:

$$
\mathbf{M}_{\mathrm{i}}^{\mathrm{e}}=\exp \left(\tau_{\mathrm{i}} \mathbf{G}_{\mathrm{i}}\right)=\mathbf{I}+\tau_{\mathrm{i}} \mathbf{G}_{\mathrm{i}}+\frac{1}{2} \tau_{\mathrm{i}}^{2} \mathbf{G}_{\mathrm{i}}^{2}+O\left(\tau_{\mathrm{i}}^{3}\right)
$$

where the term $O\left(\tau_{\mathrm{i}}^{3}\right)$ is for the terms of order higher than two.

Six generators $\mathbf{G}_{1-6}$ are the well known generators of $S O(3,1)$ the proper orthochronous Lorentz group and $\mathbf{G}_{0}$ is the identity matrix. These generators and their relations are described in Appendix A.

\section{B. Medium with fluctuating parameters}

We address now the case in which each of the values $\tau_{\mathrm{i}}$ of the elementary optical property is assumed to be temporally random and fluctuating around its mean value.

$\tau_{\mathrm{i}}$ can then be decomposed as the sum of a deterministic part and a stationary ergodic zero mean process so that:

$$
\begin{aligned}
& \tau_{\mathrm{i}}=\mu_{\mathrm{i}}+\sigma_{\mathrm{i}} \\
& \left\langle\tau_{\mathrm{i}}\right\rangle=\mu_{\mathrm{i}}\left\langle\sigma_{\mathrm{i}}\right\rangle=0
\end{aligned}
$$

Where $\left\langle\mathrm{X}\right.$. $>$ denotes the mean value of $\mathrm{X} . \sigma_{\mathrm{i}}$ is the zero mean process and the deterministic and random processes are assumed independent.

In the limit in which the thickness of the section of the medium approaches zero, it is always possible to consider $\mu_{\mathrm{i}}{ }^{2}$ negligible compared to $\mu_{\mathrm{i}}$. However, the energy of the fluctuations $<\sigma_{\mathrm{i}}^{2}>$ has not to be assumed negligible compared to $\mu_{\mathrm{i}}$ in order to take into account the effect of these fluctuations. Since $\left.<\sigma_{\mathrm{i}}\right\rangle=0$ in our model, the first significant term is $<\sigma_{\mathrm{i}}^{2}>$ when the thickness vanished. As we are dealing with passive system it is necessary to specify that the isotropic absorption coefficient has to be negative. We thus impose that $\left\langle\sigma_{0}^{2}>\right.$ is not negligible compared to $\mu_{0}$ but $\mu_{0}+<\sigma_{0}^{2}>$ remains always negative.

The Taylor series expansion of order 2 of an elementary Mueller matrix can be written as:

$$
\mathbf{M}_{\mathrm{i}}^{\mathrm{e}}=\exp \left(\tau_{\mathrm{i}} \mathbf{G}_{\mathrm{i}}\right)=\mathbf{I}+\left(\mu_{\mathrm{i}}+\sigma_{\mathrm{i}}\right) \mathbf{G}_{\mathrm{i}}+\left(\sigma_{\mathrm{i}} \mu_{\mathrm{i}}+\frac{1}{2} \sigma_{\mathrm{i}}^{2}\right) \mathbf{G}_{\mathrm{i}}^{2}+O\left(\tau_{\mathrm{i}}^{3}\right)
$$

It is worth noticing that this last hypothesis leads to one consequence. Unlike the case where the medium is deterministic $\left(\sigma_{\mathrm{i}}\right.$ $=0)$ the expression of the elementary matrix $\mathbf{M}^{\mathbf{e}}$ as a sandwich of thin laminae

$$
\mathbf{M}^{\mathrm{e}}=\prod_{\mathrm{i} \in\{0,6\}} \mathbf{M}_{\mathrm{i}}^{\mathrm{e}}
$$

is now dependent on the ordering of the laminae since the generators $\mathbf{G}_{\mathrm{i}}$ are not commuting matrices (see Appendix A). 
According to the hypothesis on $\left\{\sigma_{\mathrm{i}}, \mu_{\mathrm{i}}\right\}$ and the previous remark the mean Mueller matrix of a thin section of the medium is given from Eq. (13)-(14) by:

$$
\begin{aligned}
\left\langle\mathbf{M}^{\mathrm{e}}\right\rangle=\mathbf{I}+\sum_{\mathrm{i}=0}^{6} \mu_{\mathrm{i}} \mathbf{G}_{\mathrm{i}} & +\sum_{\substack{(\mathrm{i}, j) \in\{0,6\} \\
\mathrm{i} \neq \mathrm{j}}}\left\langle\sigma_{\mathrm{i}} \sigma_{\mathrm{j}}\right\rangle \mathbf{G}_{\mathrm{i}} \mathbf{G}_{\mathrm{j}} \\
& +\frac{1}{2} \sum_{\mathrm{i} \in\{0,6\}}\left\langle\sigma_{\mathrm{i}}^{2}\right\rangle \mathbf{G}_{\mathrm{i}}^{2}+O\left(\tau_{\mathrm{i}}^{3}\right)
\end{aligned}
$$

It is worth noticing that if all the random processes $\sigma_{\mathrm{i}}$ are assumed statistically independent. Eq. (16) becomes:

$$
\left\langle\mathbf{M}^{\mathrm{e}}\right\rangle=\mathbf{I}+\sum_{\mathrm{i}=0}^{6} \mu_{\mathrm{i}} \mathbf{G}_{\mathrm{i}}+\frac{1}{2} \sum_{\mathrm{i} \in\{0,6\}}\left\langle\sigma_{\mathrm{i}}^{2}\right\rangle \mathbf{G}_{\mathrm{i}}^{2}+O\left(\tau_{\mathrm{i}}^{3}\right)
$$

Using a classical approximation of the logarithm near the unity: $\log (\mathbf{I}+\mathbf{B})=\mathbf{B}+O\left(|\mathbf{B}|^{2}\right)$, we have:

$$
\log \left(\left\langle\mathbf{M}^{\mathrm{e}}\right\rangle\right)=\log \left(\left\langle\prod_{i \in\{0,6\}} \mathbf{M}_{\mathrm{i}}^{\mathrm{e}}\right\rangle\right) \frac{\dot{\frac{i}{j}}}{\mathfrak{j}}=\log \left(\mathbf{I}+\tau \mathbf{G}+O\left(\tau^{2}\right)\right)=\tau \mathbf{G}+O\left(\tau^{2}\|\mathbf{G}\|^{2}\right)
$$

where $\tau$ is the mean value of the thickness of the six thin laminae sandwich and

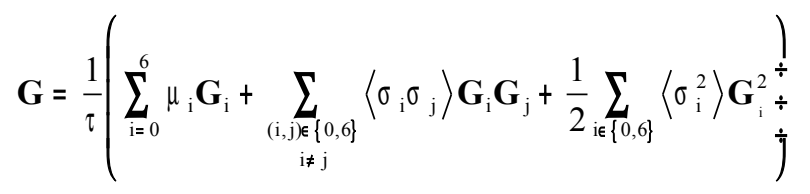

If $\tau$ approaches zero:

$$
\left\langle\mathbf{M}^{\mathrm{e}}\right\rangle=\exp \left[\tau \mathbf{G}+O\left(\tau^{2}\|\mathbf{G}\|^{2}\right)\right]
$$

A medium of thickness $z$ may be considered as a pile composed by $q$ independent statistical realizations of the previous sandwich. Since $q \tau=z$, we have from Eq. (20):

$$
\left\langle\mathbf{M}^{\mathrm{e}}\right\rangle^{q}=\exp \left[q \tau \mathbf{G}+O\left(q \tau^{2}\|\mathbf{G}\|^{2}\right)\right]=\exp \left[z \mathbf{G}+O\left(z \tau\|\mathbf{G}\|^{2}\right)\right]
$$

Thus when $\tau$ approaches zero and $q$ becomes infinite since $z$ is constant:

$$
\mathbf{M}(z)=\lim _{q \rightarrow+\infty}\left\langle\mathbf{M}^{\mathrm{e}}\right\rangle^{q}=\lim _{\tau \rightarrow 0}\left\langle\mathbf{M}^{\mathrm{e}}\right\rangle^{\frac{z}{\tau}}=\exp [z \mathbf{G}]
$$

And $\mathbf{G}$ can be written as:

$$
\mathbf{G}=\sum_{\mathrm{i}=0}^{6} \mu_{\mathrm{i}} \mathbf{G}_{\mathrm{i}}+\sum_{\substack{(\mathrm{i}, j) \in\{0,6\} \\ \mathrm{i} \neq \mathrm{j}}}\left\langle\sigma_{\mathrm{i}} \sigma_{\mathrm{j}}\right\rangle \mathbf{G}_{\mathrm{i}} \mathbf{G}_{\mathrm{j}}+\frac{1}{2} \sum_{\mathrm{i} \in\{0,6\}}\left\langle\sigma_{\mathrm{i}}^{2}\right\rangle \mathbf{G}_{\mathrm{i}}^{2}
$$

provided to define $\mu_{\mathrm{i}}$ and $\sigma_{\mathrm{i}}$ as parameters per unit length and $<\sigma_{\mathrm{i}}^{2}>$ as the energy of the fluctuations per unit length.

As we have already noted $\mathbf{G}$ takes different forms according to the order of the elements $\mathbf{G}_{\mathrm{i}}$. However, regardless of the order of $\mathbf{G}_{\mathbf{i}}$, it is always possible to write:

$$
\mathbf{G}_{\mathrm{i}} \mathbf{G}_{\mathrm{j}}=\frac{1}{2}\left\{\mathbf{G}_{\mathrm{i}}, \mathbf{G}_{\mathrm{j}}\right\}+\frac{1}{2}\left[\mathbf{G}_{\mathrm{i}}, \mathbf{G}_{\mathrm{j}}\right]
$$


Where $[\mathrm{A}, \mathrm{B}]=\mathrm{AB}-\mathrm{BA}$ is the commutator operator and $\{\mathrm{A}, \mathrm{B}\}=\mathrm{AB}+\mathrm{BA}$ is the anticommutator operator. This anticommutator operator is obviously independent of the order of the $\mathbf{G}_{\mathrm{i}}$ elements.

G can be thus decomposed in two parts:

$$
\begin{aligned}
& \mathbf{G}_{\mathbf{m}}=\sum_{\mathrm{i}=0}^{6}\left(\mu_{\mathrm{i}}+\left\langle\sigma_{0} \sigma_{\mathrm{i}}\right\rangle\right) \mathbf{G}_{\mathrm{i}}+\frac{1}{2}\left[\sum_{\substack{(\mathrm{i}, j) \in\{1,6\} \\
i \neq j}}\left\langle\sigma_{\mathrm{i}} \sigma_{j}\right\rangle\left[\mathbf{G}_{\mathrm{i}}, \mathbf{G}_{\mathrm{j}}\right]\right] \\
& \mathbf{G}_{\mathbf{u}}=\frac{1}{2}\left[\sum_{\substack{(\mathrm{i}, j) \in\{1,6\} \\
\mathbf{i} \neq \mathrm{j}}}\left\langle\sigma_{\mathrm{i}} \sigma_{j}\right\rangle\left\{\mathbf{G}_{\mathrm{i}}, \mathbf{G}_{\mathrm{j}}\right\}\right]+\sum_{\mathrm{i}=1}^{6}\left\langle\sigma_{\mathrm{i}}^{2}\right\rangle \mathbf{G}_{\mathrm{i}}^{2}
\end{aligned}
$$

It is also possible by a direct calculation of anticommutators and commutators (see Appendix A) to demonstrate that $\mathbf{G}_{\mathrm{m}}$ and $\mathbf{G}_{\mathrm{u}}$ are the Minkowski antisymmetric and symmetric components of $\mathbf{G}$ [4]. It is worth noticing that $\mathbf{G}_{\mathrm{u}}$ is independent of the order of the $\mathbf{G}_{\mathrm{i}}$ elements and then can be considered as an invariant but $\mathbf{G}_{\mathrm{m}}$ is not.

$\mathbf{G}_{\mathrm{u}}$ has the following invariant expression:

$$
\mathbf{G}_{u}=\left[\begin{array}{cccc}
\left\langle\sigma_{4}^{2}\right\rangle+\left\langle\sigma_{5}^{2}\right\rangle+\left\langle\sigma_{6}^{2}\right\rangle & -\frac{1}{2}\left(\left\langle\sigma_{2} \sigma_{6}\right\rangle-\left\langle\sigma_{3} \sigma_{5}\right\rangle\right) & -\frac{1}{2}\left(\left\langle\sigma_{3} \sigma_{4}\right\rangle-\left\langle\sigma_{1} \sigma_{6}\right\rangle\right) & -\frac{1}{2}\left(\left\langle\sigma_{1} \sigma_{5}\right\rangle-\left\langle\sigma_{2} \sigma_{4}\right\rangle\right) \\
+\frac{1}{2}\left(\left\langle\sigma_{2} \sigma_{6}\right\rangle-\left\langle\sigma_{3} \sigma_{5}\right\rangle\right) & \left\langle\sigma_{4}^{2}\right\rangle-\left\langle\sigma_{2}^{2}\right\rangle-\left\langle\sigma_{3}^{2}\right\rangle & +\frac{1}{2}\left(\left\langle\sigma_{1} \sigma_{2}\right\rangle+\left\langle\sigma_{4} \sigma_{5}\right\rangle\right) & +\frac{1}{2}\left(\left\langle\sigma_{1} \sigma_{3}\right\rangle+\left\langle\sigma_{4} \sigma_{6}\right\rangle\right) \\
+\frac{1}{2}\left(\left\langle\sigma_{3} \sigma_{4}\right\rangle-\left\langle\sigma_{1} \sigma_{6}\right\rangle\right) & +\frac{1}{2}\left(\left\langle\sigma_{1} \sigma_{2}\right\rangle+\left\langle\sigma_{4} \sigma_{5}\right\rangle\right) & \left\langle\sigma_{5}^{2}\right\rangle-\left\langle\sigma_{1}^{2}\right\rangle-\left\langle\sigma_{3}^{2}\right\rangle & +\frac{1}{2}\left(\left\langle\sigma_{2} \sigma_{3}\right\rangle+\left\langle\sigma_{5} \sigma_{6}\right\rangle\right) \\
+\frac{1}{2}\left(\left\langle\sigma_{1} \sigma_{5}\right\rangle-\left\langle\sigma_{2} \sigma_{4}\right\rangle\right) & +\frac{1}{2}\left(\left\langle\sigma_{1} \sigma_{3}\right\rangle+\left\langle\sigma_{4} \sigma_{6}\right\rangle\right) & +\frac{1}{2}\left(\left\langle\sigma_{2} \sigma_{3}\right\rangle+\left\langle\sigma_{5} \sigma_{6}\right\rangle\right) & \left\langle\sigma_{6}^{2}\right\rangle-\left\langle\sigma_{1}^{2}\right\rangle-\left\langle\sigma_{2}^{2}\right\rangle
\end{array}\right]
$$

Note that factor $\left\langle\sigma_{4}^{2}\right\rangle+\left\langle\sigma_{5}^{2}\right\rangle+\left\langle\sigma_{6}^{2}\right\rangle$ can always been subtracted from the main diagonal of $\mathbf{G}_{\mathrm{u}}$.

The expression of $\mathbf{G}_{\mathrm{m}}$ is $\mathbf{G}_{\mathrm{i}}$ order dependent however two configurations of $\mathbf{G}_{\mathrm{m}}$ will differ only by the signs of the factors $<\sigma_{i} \sigma_{j}>$ since $\left[\mathbf{G}_{i}, \mathbf{G}_{j}\right]=-\left[\mathbf{G}_{j}, \mathbf{G}_{i}\right]$. As example we give the expression of $\mathbf{G}_{\mathrm{m}}$ for the configuration $(4,5,6,1,2,3)$ of the sandwich:

$\mathbf{G}_{\mathrm{m}}=\left[\begin{array}{cccc}\mu_{0}+\left\langle\sigma_{0}^{2}\right\rangle & \mu_{4}+\left\langle\sigma_{0} \sigma_{4}\right\rangle-\frac{1}{2}\left(\left\langle\sigma_{2} \sigma_{6}\right\rangle-\left\langle\sigma_{3} \sigma_{5}\right\rangle\right) & \mu_{5}+\left\langle\sigma_{0} \sigma_{5}\right\rangle-\frac{1}{2}\left(\left\langle\sigma_{3} \sigma_{4}\right\rangle-\left\langle\sigma_{1} \sigma_{6}\right\rangle\right) & \mu_{6}+\left\langle\sigma_{0} \sigma_{6}\right\rangle-\frac{1}{2}\left(\left\langle\sigma_{1} \sigma_{5}\right\rangle-\left\langle\sigma_{2} \sigma_{4}\right\rangle\right) \\ \mu_{4}+\left\langle\sigma_{0} \sigma_{4}\right\rangle-\frac{1}{2}\left(\left\langle\sigma_{2} \sigma_{6}\right\rangle-\left\langle\sigma_{3} \sigma_{5}\right\rangle\right) & \mu_{0}+\left\langle\sigma_{0}^{2}\right\rangle & \mu_{3}+\left\langle\sigma_{0} \sigma_{3}\right\rangle+\frac{1}{2}\left(\left\langle\sigma_{4} \sigma_{5}\right\rangle-\left\langle\sigma_{1} \sigma_{2}\right\rangle\right) & -\mu_{2}-\left\langle\sigma_{0} \sigma_{2}\right\rangle+\frac{1}{2}\left(\left\langle\sigma_{4} \sigma_{6}\right\rangle-\left\langle\sigma_{1} \sigma_{3}\right\rangle\right) \\ \mu_{5}+\left\langle\sigma_{0} \sigma_{5}\right\rangle-\frac{1}{2}\left(\left\langle\sigma_{3} \sigma_{4}\right\rangle-\left\langle\sigma_{1} \sigma_{6}\right\rangle\right) & -\mu_{3}-\left\langle\sigma_{0} \sigma_{3}\right\rangle-\frac{1}{2}\left(\left\langle\sigma_{4} \sigma_{5}\right\rangle-\left\langle\sigma_{1} \sigma_{2}\right\rangle\right) & \mu_{0}+\left\langle\sigma_{0}^{2}\right\rangle & \mu_{1}+\left\langle\sigma_{0} \sigma_{1}\right\rangle+\frac{1}{2}\left(\left\langle\sigma_{5} \sigma_{6}\right\rangle-\left\langle\sigma_{2} \sigma_{3}\right\rangle\right) \\ \mu_{6}+\left\langle\sigma_{0} \sigma_{6}\right\rangle-\frac{1}{2}\left(\left\langle\sigma_{1} \sigma_{5}\right\rangle-\left\langle\sigma_{2} \sigma_{4}\right\rangle\right) & \mu_{2}+\left\langle\sigma_{0} \sigma_{2}\right\rangle-\frac{1}{2}\left(\left\langle\sigma_{4} \sigma_{6}\right\rangle-\left\langle\sigma_{1} \sigma_{3}\right\rangle\right) & -\mu_{1}-\left\langle\sigma_{0} \sigma_{1}\right\rangle-\frac{1}{2}\left(\left\langle\sigma_{5} \sigma_{6}\right\rangle-\left\langle\sigma_{2} \sigma_{3}\right\rangle\right) & \mu_{0}+\left\langle\sigma_{0}^{2}\right\rangle\end{array}\right]$

Again, the isotropic absorption coefficient $\mu_{0}+<\boldsymbol{\sigma}_{0}^{2}>$ can always be removed by subtracting $\left(\mu_{0}+<\boldsymbol{\sigma}_{0}^{2}>\right) \mathbf{I}$.

\section{PHYSICAL SIGNIFICANCE AND PROPERTIES OF DEPOLARIZING DIFFERENTIAL MUELLER MATRIX}


It is obvious that Eq. (26) and (27) are exactly Eq. (5) decomposed into a mean and uncertainty part according to Eq. (8) (meaning that $\mathbf{L}_{\mathrm{m}}=\mathbf{G}_{\mathrm{m}}$ and $\mathbf{L}_{\mathrm{u}}=\mathbf{G}_{\mathrm{u}}$ ), but we can now clarify the meaning of terms $d_{0-6}$ and $d_{7-15}$ of these two matrices.

For the $\mathbf{G}_{\mathrm{m}}$ matrix we can see that the entries $d_{0-6}$ of matrix $\mathbf{m}$ are not exactly the mean values of the elementary optical properties except if the fluctuations of the processes $\sigma_{\mathrm{i}}$ are independent (or at least non-correlated).

This reflects the fact that optically neutral medium at rest (the Mueller matrix is the identity and $\mu_{\mathrm{i}}$ are all zero) may acquire optical properties (birefringence, dichroism and attenuation) by the appearance of fluctuations into the medium.

It is also interesting to note that each term correlation in addition to the $\mu_{\mathrm{i}}$ comes from the correlation of the processes associated with complementary optical properties. The correlation between the processes related to the properties of birefringence and dichroism along the $45^{\circ}$ axes or circular is added to $\mu_{1}$ for example, which is on the linear dichroism along the $x-y$ laboratory axes. And so on.

The same is valid for the matrix $\mathbf{G}_{\mathrm{u}}$ that expresses the uncertainties of the properties. The uncertainty on the linear dichroism along the $\mathrm{x}-\mathrm{y}$ laboratory axes $\left(d_{1}\right)$ is due to the correlation between the processes related to the properties of birefringence and dichroism but along the $45^{\circ}$ axes and circular.

Being $d_{7-15}$ coefficients not independent is obvious since they are related to correlation between the different fluctuations. So put all these coefficients (with the exception of $d_{13-15}$ ) to zero except one, will result in an invalid Mueller matrix. The exception for $d_{13-15}$ comes from the case where fluctuations are not correlated.

If the fluctuations of the processes $\sigma_{\mathrm{i}}$ are non-correlated, all the off-diagonal terms of $\mathbf{G}_{\mathrm{u}}$ vanish and $\mathbf{G}_{\mathrm{u}}$ is restricted to its diagonal form $\mathbf{G}_{\mathrm{u}}{ }^{D}$ :

$$
\mathbf{G}_{\mathrm{u}}=\left[\begin{array}{cccc}
\left\langle\sigma_{4}^{2}\right\rangle+\left\langle\sigma_{5}^{2}\right\rangle+\left\langle\sigma_{6}^{2}\right\rangle & 0 & 0 & 0 \\
0 & \left\langle\sigma_{4}^{2}\right\rangle-\left\langle\sigma_{2}^{2}\right\rangle-\left\langle\sigma_{3}^{2}\right\rangle & 0 & 0 \\
0 & 0 & \left\langle\sigma_{5}^{2}\right\rangle-\left\langle\sigma_{1}^{2}\right\rangle-\left\langle\sigma_{3}^{2}\right\rangle & 0 \\
0 & 0 & 0 & \left\langle\sigma_{6}^{2}\right\rangle-\left\langle\sigma_{1}^{2}\right\rangle-\left\langle\sigma_{2}^{2}\right\rangle
\end{array}\right]
$$

The principal axes of this depolarizer are along the Poincaré representation axis (the last three Stokes parameters normalized by intensity). However, in the general case the principal axes of a depolarizer can be along any three orthogonal axes. The general expression of $\mathbf{G}_{\mathrm{u}}$ can always be diagonalized. Since such a $\mathbf{G}_{\mathrm{u}}$ matrix is completely defined by one of both the forms of $\mathrm{Eq}$ (10), the diagonal matrix $\mathbf{G}_{\mathrm{u}}{ }^{D}$ associated to $\mathbf{G}_{\mathrm{u}}$ is obviously the $\mathbf{m}^{(1)}$ expression of Eq. (11), since $\mathbf{m}^{(1)}$ is already a diagonal matrix or the diagonalized form of the $\mathbf{m}^{(2)}$ expression of Eq. (11):

$$
\begin{gathered}
\mathbf{G}_{u}^{D l}=\operatorname{diag}\left(k_{1}+k_{2}+k_{3}, k_{1}-k_{2}-k_{3}, k_{2}-k_{1}-k_{3}, k_{3}-k_{1}-k_{2}\right) \\
\text { or } \\
\mathbf{G}_{u}^{D 2}=\operatorname{diag}\left[0.5\left(k_{1}+k_{4}\right), 0.5\left(k_{1}-k_{4}\right),-0.5 k_{1},-0.5 k_{1}\right]
\end{gathered}
$$

A straightforward calculation gives the expressions of the $\left(k_{1}, k_{2}, k_{3}\right)$ or $\left(k_{1}, k_{4}\right)$ parameters from Eq. (28) and (29). For the first expression $\mathbf{G}_{\mathrm{u}}{ }^{D l}$ :

$$
\begin{aligned}
& k_{1}=\left\langle\sigma_{1}^{2}\right\rangle+\left\langle\sigma_{4}^{2}\right\rangle \\
& k_{2}=\left\langle\sigma_{2}^{2}\right\rangle+\left\langle\sigma_{5}^{2}\right\rangle \\
& k_{3}=\left\langle\sigma_{3}^{2}\right\rangle+\left\langle\sigma_{6}^{2}\right\rangle
\end{aligned}
$$

For the second expression $\mathbf{G}_{\mathrm{u}}{ }^{D 2}$ : 


$$
\begin{aligned}
& k_{1}=\left\langle\sigma_{1}^{2}\right\rangle+\left\langle\sigma_{4}^{2}\right\rangle \\
& k_{4}=\left\langle\sigma_{2}^{2}\right\rangle+\left\langle\sigma_{3}^{2}\right\rangle+\left\langle\sigma_{5}^{2}\right\rangle+\left\langle\sigma_{6}^{2}\right\rangle \\
& \text { and } \\
& 0.5\left(k_{1}+k_{4}\right)=\left\langle\sigma_{1}^{2}\right\rangle+\left\langle\sigma_{2}^{2}\right\rangle+\left\langle\sigma_{3}^{2}\right\rangle=\left\langle\sigma_{4}^{2}\right\rangle+\left\langle\sigma_{5}^{2}\right\rangle+\left\langle\sigma_{6}^{2}\right\rangle
\end{aligned}
$$

The positivity of $\left(k_{1}, k_{2}, k_{3}\right)$ or $\left(k_{1}, k_{4}\right)$ parameters of Eq. (11) is then due to their definition as sums of mean square values (or energies) of the fluctuations. The anisotropic depolarization coefficients $d_{13-15}$ can then be analyzed as composition of mean square values of linear birefringence and dichroism fluctuations along the $\mathrm{x}-\mathrm{y}$ laboratory axes $\left(k_{1}\right.$ parameter $)$, mean square values of linear birefringence and dichroism fluctuations along the $45^{\circ}$ axes $\left(k_{2}\right.$ and $k_{4}$ parameters $)$ and mean square values of circular birefringence and dichroism fluctuations $\left(k_{3}\right.$ and $k_{4}$ parameters). It is worth noticing that the $\left(k_{1}, k_{4}\right)$ parameters model is related to a configuration with identical mean square value of dichroism and birefringence fluctuations.

These results can be illustrated on the example already analyzed in [4] of an experimental Mueller matrix, measured by Gosh $\&$ al. [15] from a biological phantom in a transmission experiment. The medium consists of a dispersion of polystyrene microspheres in a sucrose-containing polymerized polyacrylamide and exhibits properties of linear, circular birefringence, and turbidity (depolarizing).

$$
\mathrm{M}_{\text {phantom }}=\left[\begin{array}{cccc}
1 & 0.0185 & 0.0029 & 0.0042 \\
0.0172 & 0.7569 & -0.0405 & 0.0462 \\
0.0034 & 0.0524 & 0.5450 & -0.5466 \\
0.0024 & -0.0070 & 0.6244 & 0.5967
\end{array}\right]=\mathrm{M}_{\Delta} \mathrm{M}_{\mathrm{R}} \mathrm{M}_{\mathrm{D}}
$$

The corresponding decomposed depolarizing $\mathbf{M}_{\Delta}$, retardance $\mathbf{M}_{R}$ and diattenuation $\mathbf{M}_{\mathrm{D}}$ matrices obtained from the Lu and Chipman decomposition [ ] are given by:

$$
\mathrm{M}_{\Delta}=\left[\begin{array}{cccc}
1 & 0 & 0 & 0 \\
0.0031 & 0.7593 & -0.0050 & -0.0019 \\
0.0031 & -0.0050 & 0.7737 & 0.0084 \\
-0.0018 & -0.0019 & 0.0084 & 0.8638
\end{array}\right] \mathrm{M}_{\mathrm{R}}=\left[\begin{array}{cccc}
1 & 0 & 0 & 0 \\
0 & 0.9972 & -0.0470 & 0.0578 \\
0 & 0.0742 & 0.6964 & -0.7138 \\
0 & -0.0067 & 0.7161 & 0.6980
\end{array}\right] \mathrm{M}_{\mathrm{D}}=\left[\begin{array}{cccc}
1 & 0.0185 & 0.0029 & 0.0042 \\
0.0185 & 1 & 0 & 0 \\
0.0029 & 0 & 0.9998 & 0 \\
0.0042 & 0 & 0 & 0.9998
\end{array}\right]
$$

It is not a question of comparing the results of this decomposition with those of the differential approach. The underlying

\begin{tabular}{|c|c|c|c|c|c|c|c|c|c|}
\hline $\mathbf{X}$ & \multicolumn{4}{|c|}{$\mathbf{L}_{\mathrm{m}}(\mathbf{X})$} & \multicolumn{4}{|c|}{$\mathbf{L}_{\mathrm{u}}(\mathbf{X})$} & $\mathrm{k}_{\mathrm{i}}(\mathbf{X})$ \\
\hline \multirow{4}{*}{$\mathbf{M}_{\Delta}$} & 0 & 0.0018 & 0.001 & -0.001 & 0 & -0.0018 & -0.0018 & 0.001 & \\
\hline & 0.0018 & 0 & 0 & 0 & 0.0018 & -0.275 & -0.0065 & -0.0023 & 0.1938 \\
\hline & 0.0018 & 0 & 0 & 0 & 0.0018 & -0.0065 & -0.2566 & 0.0103 & $\mathrm{k}_{2}=0.0836$ \\
\hline & -0.001 & 0 & 0 & 0 & -0.001 & -0.002 & 0.0103 & -0.1465 & $\mathrm{k}_{3}=0.0617$ \\
\hline \multirow{4}{*}{$\mathbf{M}_{\Delta} \mathbf{M}_{\mathrm{D}}$} & 0 & 0.0204 & 0.004 & 0.0032 & 0 & 0.0008 & -0.001 & 0.0013 & \\
\hline & 0.0204 & 0 & 0 & 0 & -0.0008 & -0.275 & -0.006 & -0.0023 & \\
\hline & 0.0047 & 0 & 0 & 0 & 0.0014 & -0.006 & -0.256 & 0.0103 & $\mathrm{k}_{2}=0.0836$ \\
\hline & 0.0032 & 0 & 0 & 0 & -0.0013 & -0.002 & 0.0103 & -0.1464 & 617 \\
\hline
\end{tabular}
physical models are not the same. The turbidity of the medium is assumed to be spatially localized for instance in the Lu and Chipman decomposition. This decomposition only serves us to generate Mueller matrices related to media with different types of optical properties.

Tab.1 Mueller matrices and corresponding $\mathbf{L}_{\mathrm{m}}$ and $\mathbf{L}_{\mathrm{u}}$ matrices. The $\mathrm{k}_{\mathrm{i}}$ coefficients calculated from $\mathbf{L}_{\mathrm{u}}$ matrices are also presented. 


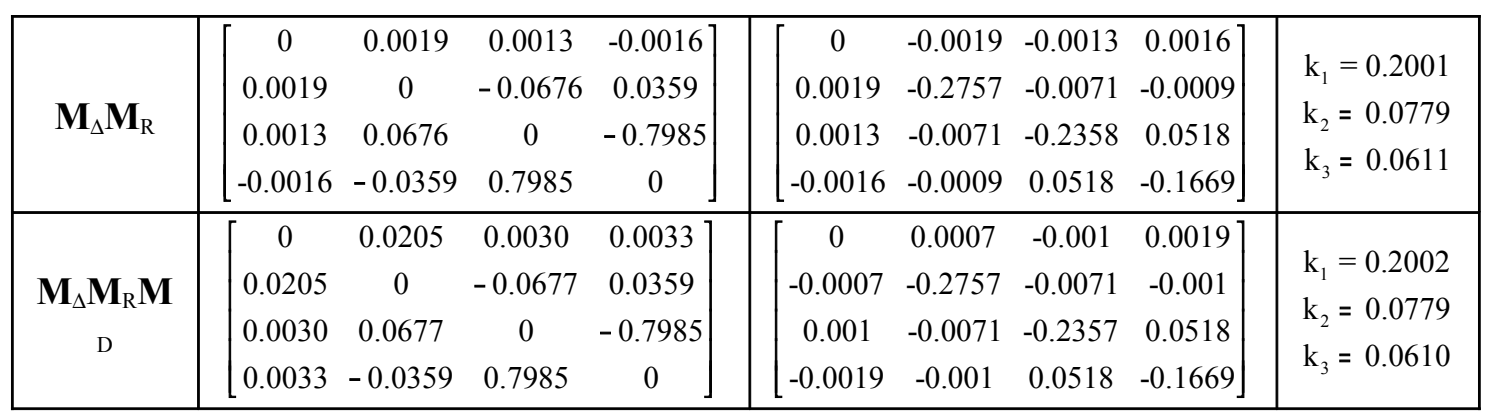

We first consider the case of $\mathbf{M}_{\Delta}$ alone. This matrix accounts for the depolarizing effects related to the turbidity of the medium. Tab. 1 gives the corresponding $\mathbf{L}_{\mathrm{m}}$ and $\mathbf{L}_{\mathrm{u}}$ matrices. All the off-diagonal entries of $\mathbf{L}_{\mathrm{m}}$ are null or identical to corresponding entries of $\mathbf{L}_{\mathrm{u}}$. From Eq. (26) and (27) it is thus immediate to deduce that the $\mu_{1-6}$ values must be zero (bearing in mind that two configurations of $\mathbf{G}_{\mathrm{m}}$ will differ only by the signs of the factors $<\sigma_{\mathrm{i}} \sigma_{\mathrm{j}}>$ ). The entries values of $\mathbf{L}_{\mathrm{m}}$ are thus exclusively due to fluctuations. This result is consistent with the definition of $\mathbf{M}_{\Delta}$ as characterizing the purely depolarizing part of the medium. Since $\mathrm{k}_{1}$ is much larger than $\mathrm{k}_{2}$ and $\mathrm{k}_{3}$, we can conclude from Eq. (30) that the linear birefringence and (or) dichroism along the $\mathrm{x}-\mathrm{y}$ laboratory axes are the optical properties which mainly fluctuate in this medium.

We now consider the matrix $\mathbf{M}_{\Delta} \mathbf{M}_{\mathrm{D}}$. This matrix accounts for the depolarizing and diattenuation (or dichroism) effects of the medium. From the corresponding $\mathbf{L}_{\mathrm{m}}$ and $\mathbf{L}_{\mathrm{u}}$ matrices (given Tab. 1) we see immediately that there is no increase in the birefringence since the corresponding entries of $\mathbf{L}_{m}\left(\mathbf{M}_{\Delta} \mathbf{M}_{\mathrm{D}}\right.$ ) are null ( $\mu_{1-3}$ values are then null and there is no contribution from the fluctuations) and the corresponding sub-matrices of $\mathbf{L}_{\mathrm{u}}\left(\mathbf{M}_{\Delta}\right)$ and $\mathbf{L}_{\mathrm{u}}\left(\mathbf{M}_{\Delta} \mathbf{M}_{\mathrm{D}}\right)$ are identical. Fluctuation of the birefringence only comes from $\mathbf{M}_{\Delta}$. From $\mathbf{M}_{\Delta}$ to $\mathbf{M}_{\Delta} \mathbf{M}_{\mathrm{D}}$ the total energy of the fluctuations and its distribution are unchanged ( $\mathrm{k}_{\mathrm{i}}$ values are identical for both matrices). With the exception of the first column and the first row, all other entries of $\mathbf{L}_{\mathrm{u}}$ remain unchanged. As a result, the changes in the coefficients values of the first column of $\mathbf{L}_{m}\left(\mathbf{M}_{\Delta} \mathbf{M}_{\mathrm{D}}\right)$ are due solely to the matrix $\mathbf{M}_{\mathrm{D}}$. This can be verified by subtracting the values of the first column of $\mathbf{L}_{\mathrm{m}}\left(\mathbf{M}_{\Delta}\right)$ to the values of the first column of $\mathbf{L}_{\mathrm{m}}\left(\mathbf{M}_{\Delta} \mathbf{M}_{\mathrm{D}}\right)$. We recover the values of the first column of $\mathbf{M}_{\mathrm{D}}$.

From $\mathbf{M}_{\Delta}$ to $\mathbf{M}_{\Delta} \mathbf{M}_{\mathrm{R}}$ the total energy of the fluctuations is unchanged $\left(\mathrm{k}_{1}+\mathrm{k}_{2}+\mathrm{k}_{3}\right.$ has the same value in both cases) but its distribution is changed. First column entries of $\mathbf{L}_{\mathrm{m}}\left(\mathbf{M}_{\Delta} \mathbf{M}_{\mathrm{R}}\right)$ and $\mathbf{L}_{\mathrm{u}}\left(\mathbf{M}_{\Delta} \mathbf{M}_{\mathrm{R}}\right)$ are identical. This means again that these coefficients (related to dichroism) are only due to fluctuations ( $\mu_{46}$ values are then null). This corresponds well with the fact that $\mathbf{M}_{R}$ does not contribute to the dichroism in the resulting product $\mathbf{M}_{\Delta} \mathbf{M}_{\mathrm{R}}$. Nevertheless the values are different from those of $\mathbf{L}_{\mathrm{u}}\left(\mathbf{M}_{\Delta}\right)$ which matches the fact that $\mathbf{M}_{\mathrm{R}}$ modifies fluctuations of birefringence coming from $\mathbf{M}_{\Delta}$.

We now consider the matrix $\mathbf{M}_{\Delta} \mathbf{M}_{\mathrm{R}} \mathbf{M}_{\mathrm{D}}$. This matrix accounts for the depolarizing, diattenuation (or dichroism) and birefringence effects of the medium. The phantom is built to exhibit properties of birefringence and chirality. Polystyrene microspheres are added resulting in a scattering effect responsible for the depolarizing nature of the medium (see [] for more details).

The corresponding $\mathbf{L}_{\mathrm{m}}$ and $\mathbf{L}_{\mathrm{u}}$ matrices can be found in [4] and are recalled Tab. 1. The total energy of the fluctuations $\left(\mathrm{k}_{1}+\mathrm{k}_{2}+\mathrm{k}_{3}\right)$ keeps the same value as in the case of $\mathbf{M}_{\Delta}$ alone and the same distribution as $\mathbf{M}_{\Delta} \mathbf{M}_{\mathrm{R}}$ (an additional multiplication by $\mathbf{M}_{\mathrm{D}}$ does not change this result as we have seen). The birefringence corresponding entries of $\mathbf{L}_{\mathrm{m}}\left(\mathbf{M}_{\Delta} \mathbf{M}_{\mathrm{R}} \mathbf{M}_{\mathrm{D}}\right)$ and their fluctuations depicted in $\mathbf{L}_{\mathrm{u}}\left(\mathbf{M}_{\Delta} \mathbf{M}_{\mathrm{R}} \mathbf{M}_{\mathrm{D}}\right)$ are completely defined by $\mathbf{M}_{\Delta} \mathbf{M}_{\mathrm{R}}$ and unaffected by the additional multiplication by $\mathbf{M}_{\mathrm{D}}$ as expected.

The estimated values for the linear birefringence (and its fluctuation) $\delta=0.7985 \pm 0.0518$ and for the circular birefringence $0.0677 \pm 0.0071$ (or the associated optical rotation angle $\psi$ ) are in close agreement with the expected values as it was already noted in [4]. It was also noted small values for the linear dichroism $(0.0205 \pm 0.007)$ and linear birefringence along the $45^{\circ}$ axes $(0.0359 \pm 0.001)$. How can explain the appearance of these quantities?

As reported in [15] the medium exhibits linear birefringence inherited from strain applied along the vertical direction and chirality inherits from the concentration of sucrose. The path length of photons randomly increased due to multiple scattering effects in this birefringent-chiral medium. This random variation of path length results in a random variation of the linear and circular birefringence (i.e. by the existence of terms $\sigma_{1}$ and $\sigma_{3}$ ). These fluctuations are correlated since they come from the 
same variation in the length of the path traveled by the photon. The result is a non-zero value for the term $<\sigma_{1} \sigma_{3}>$ which occurs in the linear birefringence along the $45^{\circ}$ axes coefficient (see Eq. (27)).

The scattering-induced diattenuation is due mainly to the weakly scattered photons [17]. This effect is even more important in the backscattering geometry as compared to forward scattering geometry. As noted in [18], this scattering-induced diattenuation effect is not exhibited in a completely distributed fashion leading to an equivalent diattenuation parameter in the differential matrix model. The corresponding values of the entries of $\mathbf{L}_{\mathrm{m}}\left(\mathbf{M}_{\Delta} \mathbf{M}_{\mathrm{R}} \mathbf{M}_{\mathrm{D}}\right)$ and $\mathbf{L}_{\mathrm{u}}\left(\mathbf{M}_{\Delta} \mathbf{M}_{\mathrm{R}} \mathbf{M}_{\mathrm{D}}\right)$ clearly show that this parameter is seen as an intrinsic diattenuation parameter ( $\mu_{4}$ parameter in Eq. (27) ) and is not related to fluctuations (see the comparison above between $\mathbf{L}_{\mathrm{m}}\left(\mathbf{M}_{\Delta}\right)$ and $\left.\mathbf{L}_{\mathrm{m}}\left(\mathbf{M}_{\Delta} \mathbf{M}_{\mathrm{D}}\right)\right)$.

We can conclude from the value of the $\mathrm{k}_{1}$ parameter and Eq. (30) that the linear birefringence and (or) dichroism along the $\mathrm{x}-\mathrm{y}$ laboratory axes are the optical properties which mainly fluctuate in this medium. This result is in perfect agreement with the above discussion on the scattering effects in a turbid and birefringent medium.

\section{CONCLUSION}

A physical model of differential Mueller matrix for depolarizing uniform media has been introduced in order to address the question of significance of the parameters of differential Mueller matrix formalism. The concept of mean value and uncertainty of the optical properties recently introduced to depict this differential matrix is straightforwardly related to the random fluctuations of these optical properties. We extend the scope of the depolarizing Mueller calculus to parallel that established by Jones for his calculus based on the layered-medium interpretation. Based on the random Mueller-Jones matrix approach, the obtained parameterization perfectly fits the previous results of the literature. Necessary conditions of positivity on specific coefficients imposed in order to have physical Mueller matrix are introduced in a natural way and not inferred a posteriori. Interpretations of the underlying physical processes are also presented.

It may be argue that the previous results are only obtained for a model where the properties are assumed to be temporally random and do not cover the classical approach of depolarizing Mueller matrices as sum of Mueller-Jones matrices (nondepolarizing matrices). The answer is given by the hypothesis of ergodicity on the temporal processes. For ergodic processes all the temporal moments are equal to the statistical moments. These statistical moments are obtained by computing the moments on all the possible events of the process. According to our model, one event is just a non-depolarizing matrix obtained for one realization of the elementary properties. These statistical moments are then related to a model of depolarizing matrix defined by a sum of random realizations of non-depolarizing matrices. Extending the results to the classical approach of depolarizing Mueller matrix as sum of non-depolarizing ones is thus allowed by the ergodicity hypothesis.

\section{Acknowledgments}

The author would like to thank Julien Fade because the idea underlying this article emerged after a discussion with him.

\section{APPENDIX A}

A description of the six generators $\mathbf{G}_{1-6}$ is presented using the usual notation for generators of $S O(3,1)$ the proper orthochronous Lorentz group. The generators are dissociated in terms of more familiar quantities: 3 rotation generators $\mathrm{J}_{1}, \mathrm{~J}_{2}, \mathrm{~J}_{3}$ and 3 boost generators $\mathrm{K}_{1}, \mathrm{~K}_{2}$ and $\mathrm{K}_{3}$ :

$$
\begin{aligned}
& \mathbf{J}_{1}=\mathrm{i} \mathbf{G}_{1}=\left[\begin{array}{cccc}
0 & 0 & 0 & 0 \\
0 & 0 & 0 & 0 \\
0 & 0 & 0 & -\mathrm{i} \\
0 & 0 & \mathrm{i} & 0
\end{array}\right] \mathbf{J}_{2}=\mathrm{i \mathbf {G } _ { 2 }}=\left[\begin{array}{cccc}
0 & 0 & 0 & 0 \\
0 & 0 & 0 & \mathrm{i} \\
0 & 0 & 0 & 0 \\
0 & -\mathrm{i} & 0 & 0
\end{array}\right] \mathbf{J}_{3}=\mathrm{i} \mathbf{G}_{3}=\left[\begin{array}{cccc}
0 & 0 & 0 & 0 \\
0 & 0 & -\mathrm{i} & 0 \\
0 & \mathrm{i} & 0 & 0 \\
0 & 0 & 0 & 0
\end{array}\right] \\
& \mathbf{K}_{1}=\mathbf{i G}_{4}=\left[\begin{array}{llll}
0 & \mathrm{i} & 0 & 0 \\
\mathrm{i} & 0 & 0 & 0 \\
0 & 0 & 0 & 0 \\
0 & 0 & 0 & 0
\end{array}\right] \mathbf{K}_{2}=\mathbf{i G}_{5}=\left[\begin{array}{cccc}
0 & 0 & \mathrm{i} & 0 \\
0 & 0 & 0 & 0 \\
\mathrm{i} & 0 & 0 & 0 \\
0 & 0 & 0 & 0
\end{array}\right] \mathbf{K}_{3}=\mathbf{i G}_{6}=\left[\begin{array}{llll}
0 & 0 & 0 & \mathrm{i} \\
0 & 0 & 0 & 0 \\
0 & 0 & 0 & 0 \\
\mathrm{i} & 0 & 0 & 0
\end{array}\right]
\end{aligned}
$$


With this notation the commutators are given by:

$$
\begin{aligned}
& {\left[\mathbf{J}_{\mathrm{m}}, \mathbf{J}_{\mathrm{n}}\right]=-\mathrm{i} \alpha_{\mathrm{mnk}} \mathbf{J}_{\mathrm{k}}} \\
& {\left[\mathbf{K}_{\mathrm{m}}, \mathbf{K}_{\mathrm{n}}\right]=\mathrm{i} \alpha_{{ }_{\mathrm{mnk}}} \mathbf{K}_{\mathrm{k}}} \\
& {\left[\mathbf{J}_{\mathrm{m}}, \mathbf{K}_{\mathrm{n}}\right]=-\mathrm{i} \alpha_{m \mathrm{mk}} \mathbf{J}_{\mathrm{k}}}
\end{aligned}
$$

where $\alpha_{m n k}$ is the totally anti-symmetric second rank tensor.

The anti-commutators are given by:

$$
\begin{aligned}
& \left\{\mathbf{J}_{1}, \mathbf{J}_{1}\right\}=\left[\begin{array}{cccc}
0 & 0 & 0 & 0 \\
0 & 0 & 0 & 0 \\
0 & 0 & 2 & 0 \\
0 & 0 & 0 & 2
\end{array}\right]\left\{\mathbf{K}_{1}, \mathbf{K}_{1}\right\}=\left[\begin{array}{cccc}
-2 & 0 & 0 & 0 \\
0 & -2 & 0 & 0 \\
0 & 0 & 0 & 0 \\
0 & 0 & 0 & 0
\end{array}\right] \\
& \left\{\mathbf{J}_{2}, \mathbf{J}_{2}\right\}=\left[\begin{array}{cccc}
0 & 0 & 0 & 0 \\
0 & 2 & 0 & 0 \\
0 & 0 & 0 & 0 \\
0 & 0 & 0 & 2
\end{array}\right]\left\{\mathbf{K}_{2}, \mathbf{K}_{2}\right\}=\left[\begin{array}{cccc}
-2 & 0 & 0 & 0 \\
0 & 0 & 0 & 0 \\
0 & 0 & -2 & 0 \\
0 & 0 & 0 & 0
\end{array}\right] \\
& \left\{\mathbf{J}_{3}, \mathbf{J}_{3}\right\}=\left[\begin{array}{cccc}
0 & 0 & 0 & 0 \\
0 & 2 & 0 & 0 \\
0 & 0 & 0 & 0 \\
0 & 0 & 0 & 2
\end{array}\right]\left\{\mathbf{K}_{3}, \mathbf{K}_{3}\right\}=\left[\begin{array}{cccc}
-2 & 0 & 0 & 0 \\
0 & 0 & 0 & 0 \\
0 & 0 & 0 & 0 \\
0 & 0 & 0 & -2
\end{array}\right] \\
& \left\{\mathbf{J}_{1}, \mathbf{J}_{2}\right\}=\left\{\mathbf{K}_{1}, \mathbf{K}_{2}\right\}=\left[\begin{array}{cccc}
0 & 0 & 0 & 0 \\
0 & 0 & -1 & 0 \\
0 & -1 & 0 & 0 \\
0 & 0 & 0 & 0
\end{array}\right]\left\{\mathbf{K}_{1}, \mathbf{J}_{2}\right\}=-\left\{\mathbf{K}_{2}, \mathbf{J}_{1}\right\}=\left[\begin{array}{cccc}
0 & 0 & 0 & 1 \\
0 & 0 & 0 & 0 \\
0 & 0 & 0 & 0 \\
-1 & 0 & 0 & 0
\end{array}\right] \\
& \left\{\mathbf{J}_{1}, \mathbf{J}_{3}\right\}=\left\{\mathbf{K}_{1}, \mathbf{K}_{3}\right\}=\left[\begin{array}{cccc}
0 & 0 & 0 & 0 \\
0 & 0 & 0 & -1 \\
0 & 0 & 0 & 0 \\
0 & -1 & 0 & 0
\end{array}\right]\left\{\mathbf{K}_{1}, \mathbf{J}_{3}\right\}=-\left\{\mathbf{K}_{3}, \mathbf{J}_{1}\right\}=\left[\begin{array}{cccc}
0 & 0 & -1 & 0 \\
0 & 0 & 0 & 0 \\
1 & 0 & 0 & 0 \\
0 & 0 & 0 & 0
\end{array}\right] \\
& \left\{\mathbf{J}_{2}, \mathbf{J}_{3}\right\}=\left\{\mathbf{K}_{2}, \mathbf{K}_{3}\right\}=\left[\begin{array}{cccc}
0 & 0 & 0 & 0 \\
0 & 0 & 0 & 0 \\
0 & 0 & 0 & -1 \\
0 & 0 & -1 & 0
\end{array}\right]\left\{\mathbf{K}_{2}, \mathbf{J}_{3}\right\}=-\left\{\mathbf{K}_{3}, \mathbf{J}_{2}\right\}=\left[\begin{array}{cccc}
0 & 1 & 0 & 0 \\
-1 & 0 & 0 & 0 \\
0 & 0 & 0 & 0 \\
0 & 0 & 0 & 0
\end{array}\right]
\end{aligned}
$$

The result of anti-commutation operator is not shown when it is equal to null matrix.

\section{References}

1. R.C. Jones, "A new calculus for the treatment of optical systems. VII properties of the N-matrices", J. Opt. Soc. Am. 38, 671-685 (1948)

2. R. M. A. Azzam, "Propagation of partially polarized light through anisotropic media with or without depolarization: A differential 4x4 matrix calculus", J. Opt. Soc. Am. 68, 1756-1767 (1978)

3. . R. Barakat, "Exponential versions of the Jones and Mueller-Jones polarization matrices", J. Opt. Soc. Am. A 13, 158163 (1996)

4. R. Ossikovski, "Differential matrix formalism for depolarizing anisotropic media," Opt. Letters. 36, 2330-2332 (2011)

5. N. Ortega-Quijano, J.L. Arce-Diego, "Mueller matrix differential decomposition," Opt. Letters 36, 1942-1944 (2011). 
6. N. Ortega-Quijano, J.L. Arce-Diego, "Depolarizing differential Mueller matrices," Opt. Letters 36, 2429-2431 (2011).

7. A. Chipman, Handbook of Optics, 3rd ed., (McGraw Hill, 2009) Vol. 1.

8. H.D. Noble, R.A. Chipman, "Mueller matrix roots algorithm and computational considerations," Opt. Express 20, 17-31 (2012)

9. R. Ossikovski, "Differential and product Mueller matrix decompositions: a formal comparison," Opt. Letters 37, 220$222(2012)$

10. T. Germer, "Realizable differential matrices for depolarizing media," Opt. Letters 37, 921-923 (2012)

11. . B. N. Simon, S. Simon, N. Mukunda, F. Gori, M. Santarsiero, R. Borghi, and R. Simon, "A complete characterization of pre-Mueller and Mueller matrices in polarization optics," J. Opt. Soc. Am. A 27, 188-199 (2010)

12. V. Devlaminck, P. Terrier and J.M. Charbois," "Differential matrix physically admissible for depolarizing media: the case of diagonal matrices," Opt. Letters. 38, 1497-1499 (2013)

13. V. Devlaminck, P. Terrier and J.M. Charbois, "A physically admissible parameterization for differential Mueller matrix of uniform media," Opt. Letters 38, 1410-1412 (2013)

14. N. Ortega-Quijano, B. Haj-Ibrahim, E. García-Caurel, J. Arce-Diego, and R. Ossikovski, "Experimental validation of Mueller matrix differential decomposition," Opt. Express 20, 1151-1163 (2012)

15. N. Ghosh, M. F. G. Wood, S. Li, R. D. Weisel, B. C. Wilson, R. Li, and I. A. Vitkin, "Mueller matrix decomposition for polarized light assessment of biological tissues," J. Biophoton. 2, 145-156 (2009)

16. S. Y. Lu, R. A. Chipman, "Interpretation of Mueller matrices based on polar decomposition," J. Opt. Soc. Am. A 13, 1106-1113 (1996)

17. S. Manhas, M. K. Swami, P. Buddhiwant, N. Ghosh, P. K. Gupta, and K. Singh, “ Mueller matrix approach for determination of optical rotation in chiral turbid media in backscattering geometry," Opt. Express 14, 190-202 (2006)

18. Satish Kumar, Harsh Purwar, Razvigor Ossikovski, I . Alex Vitkin and Nirmalya Ghosh, "Comparative study of differential matrix and extended polar decomposition formalisms for polarimetric characterization of complex tissuelike turbid media,” J. Biomed. Opt. 17, 105006-1-12 (2012) 